\title{
Tempora mutantur - Aktualisierte Empfehlungen zur Behandlung der Immunthrombozytopenie
}

\author{
Axel Matzdorff \\ Klinik für Hämatologie und Onkologie, Caritasklinikum Saarbrücken St. Theresia, Deutschland
}

Die Immunthrombozytopenie (ITP) ist selten und zählt zu den «Orphan Diseases» (engl. orphan = Waise). Deshalb gibt es nur wenige in randomisierten Studien geprüfte Erkenntnisse mit hohem Evidenzgrad. Viele Therapieentscheidungen beruhen auf der persönlichen Erfahrung des einzelnen Arztes. Um diese Situation zu verbessern publizierte 1996 eine Expertengruppe der American Society of Hematology die erste Praxis-Leitlinie zur ITP [1]. Diese «ASH-Leitlinie» definierte für viele Jahre den Standard in der Diagnostik und Therapie der ITP.

Seither hat sich viel verändert. Neue Studien haben unsere Vorstellungen von der Pathophysiologie der ITP verändert. Sie ist nicht mehr nur eine Erkrankung mit verstärktem Thrombozytenabbau, auch die Thrombozytenneubildung ist gestört. Regulatorische T- und B-Lymphozyten spielen eine wichtige Rolle bei der Entstehung und Aufrechterhaltung der fehlgeleiteten Immunantwort. Neue Wirkstoffe wurden entwickelt und seit der Marktzulassung der ThrombopoetinRezeptor-Agonisten kann man von einer neuen Ära in der Behandlung der ITP sprechen. Um dieser rasanten Entwicklung Rechnung zu tragen, wurden 2010 die neuen deutschsprachigen Empfehlungen zur Diagnostik und Therapie der ITP publiziert [2]. Seither sind in PubMed wieder über 1500 Artikel zum Thema erschienen (http://www.ncbi.nlm.nih.gov/ pubmed, Suchbegriff «ITP», Stand 11/2013). Eine Aktualisierung wurde notwendig, diesmal verfasst von einer gemeinsamen Expertengruppe der Deutschen und Österreichischen Gesellschaften für Hämatologie und Medizinische Onkologie (DGHO, OeGHO), der Schweizerischen Gesellschaft für Hämatologie (SGH+SSH) sowie der Gesellschaft für Pädiatrische Onkologie und Hämatologie (GPOH).

Helfen Leitlinien uns Ärzten wirklich? Das Leitlinienregister der AWMF (Arbeitsgemeinschaft der Wissenschaftlichen Medizinischen Fachgesellschaften e.V.) listet rund 700 Publikationen, davon 123 der Klasse S3 und 159 der Klasse S2
(Stand 11/2013). Dies spricht für ein großes Interesse an Leitlinien. Eine positive Einstellung bedeutet jedoch nicht, dass Leitlinien auch angewendet werden [3]. Studien bescheinigen eine noch unzureichende Umsetzung in der täglichen Praxis $[4,5]$. Woran liegt das? Sind Leitlinien zu umfangreich? Sind sie praxisfern, zu schwer zugänglich, zu akademisch [6]?

Es war das Anliegen der Autoren, diese ITP-Leitlinie «aus der Praxis» und «für die Praxis» zu formulieren. Gliederung, Inhalt und Sprache orientieren sich an den zahlreichen Anfragen von Kollegen und Patienten bei den Autoren. Die Deutsche Gesellschaft für Hämatologie hat die neue ITP-Leitlinie auf ihre Homepage gestellt (www.onkopedia.de), um sie für alle Interessierten leicht zugänglich zu machen. Onkopedia hat über 70000 Besucher pro Monat, überwiegend Hämatologen und Onkologen. Dankenswerterweise hat sich der Karger-Verlag bereit erklärt, die Empfehlungen zusätzlich in gedruckter Version zur Verfügung zu stellen. Die Autoren hoffen, dass dadurch alle mit ITP-Patienten befassten Fachdisziplinen und Ärzte noch besser erreicht werden.

Wie hilft die neue ITP-Leitlinie unseren Patienten? Leitlinien richten sich primär an Ärzte und Wissenschaftler. Viele Alltagsprobleme der Patienten in Schule und Beruf, in der Familie, beim Abschluss von Versicherungen, bei FreizeitAktivitäten, Sport und Reisen werden nur am Rande angesprochen. Nicht wenige ITP-Patienten fühlen sich mit ihren Fragen «wie ein Waisenkind» alleine gelassen. Der Begriff Orphan Disease hat so für Sie eine eigene, neue Bedeutung bekommen. Deshalb organisieren sie sich in Selbsthilfegruppen. Darüber hinaus äußern Patienten den Eindruck, dass Kostenerwägungen bei Therapieempfehlungen eine Rolle spielen könnten. Auch der Internationale ITP-ConsensusReport von 2010 schreibt, dass bei den Empfehlungen die finanziellen Rahmenbedingungen eines Gesundheitssystems und die regulatorischen Vorgaben berücksichtigt werden mussten. Die Autoren der vorliegenden Empfehlungen orien-

\section{KARGER \\ Fax +497614520714 \\ Information@Karger.com}

www.karger.com (c) 2014 S. Karger GmbH, Freiburg $2296-5270 / 14 / 3714-0003 \$ 39.50 / 0$

Accessible online at: www.karger.com/ort 
tieren sich primär an dem ärztlich und medizinisch Sinnvollen. $\mathrm{Ob}$ eine Therapie in Deutschland, Österreich oder der Schweiz zugelassen oder «off-label» ist, wurde separat aufgelistet, ist aber nicht empfehlungsbestimmend.

Tempora mutantur nos et mutamur in illis. - Leitlinien bemühen sich um größtmögliche Objektivität und Wissenschaftlichkeit, sie sind aber immer auch ein Produkt ihrer Zeit. Immer mehr ITP-Patienten organisieren sich in Selbsthilfegruppen, um Informationen besser auszutauschen und bei gesundheitspolitischen Entscheidungen mitzuwirken. In England und den USA werden Vertreter der ITP-Selbsthilfegruppen bei der Leitlinienerstellung bereits aktiv beteiligt. Dies wäre eine Aufgabe für die nächste deutschsprachige Leitlinie oder - frei nach Sepp Herberger - nach der Leitlinie ist vor der Leitlinie.

\section{Disclosure Statement}

Der Autor gibt als potenzielle Interessenkonflikte an: Beratungstätigkeit: AMGEN, Baxter, GlaxoSmithKline; Besitz von Geschäftsanteilen, Aktien oder Fonds: Roche-Aktien in Familienbesitz; Referentenhonorare: AMGEN, Behring, GlaxoSmithKline, Roche; Finanzierung wissenschaftlicher Untersuchungen: Roche; Gutachtertätigkeit: AOK; Mitarbeiterweiterbildungen, Reisekosten, etc.: GSK, Roche, AMGEN.

\section{Literatur}

1 George JN, Woolf SH, Raskob GE, et al. Idiopathic thrombocytopenic purpura: a practice guideline developed by explicit methods for the American Society of Hematology. Blood 1996;88:3-40.

2 Matzdorff A, Giagounidis A, Greinacher A, et al. Diagnostik und Therapie der Immunthrombozytopenie. Empfehlungen einer gemeinsamen Expertengruppe der DGHO, DGTI und GTH. Onkologie 2010;33(suppl 3):2-20.
Hayward R, Guyatt G, Moore K, et al.: Canadian physicians' attitudes about preferences regarding clinical practice guidelines. CMAJ 1997;156:17151723.

4 Rogenhofer S, Zengerling F, Schrader M, Müller SC: Leitlinien: Realität und Qualität. Urologe 2012;51:1074-1077.
Karbach U, Schubert I, Hagemeister J, et al.: Ärztliches Leitlinienwissen und die Leitliniennähe hausärztlicher Therapien. Dtsch Ärztebl Int 2011; 108:61-69.

6 Nast A, Sporbeck B, Jacobs A, et al.: Wahrnehmung der Verbindlichkeit von Leitlinienempfehlungen: Eine Umfrage zu häufigen Formulierungen. Dtsch Ärztebl Int 2013;110:663-668. 